\title{
葡萄劣变过程中挥发性物质的 FTIR 光谱分析
}

\author{
王文重 ${ }^{a, b}$ 董大明*, $a$ 郑文刚 $a$ 韩峻峰 ${ }^{b}$
叶松 ${ }^{b}$ 矫雷子 $a$ 赵贤德 ${ }^{a}$ \\ ( ${ }^{a}$ 北京市农林科学院 国家农业信息化工程技术研究中心 北京 100097) \\ ( ${ }^{b}$ 桂林电子科技大学电子工程与自动化学院 桂林 541004)
}

\begin{abstract}
摘要 葡萄在运输和咜藏过程中极易发生变质, 对葡萄劣变进行预警可有效降低大规模腐败的风险. 研究了葡萄在劣 变过程中所产生挥发性物质的 FTIR 光谱特性. 实验证明了葡萄劣变中挥发性物质的主要成分为乙酸乙酯、乙醇、二氧 化碳和水汽. 通过光谱定量化分析研究挥发性物质在劣变中的变化规律, 发现葡萄在劣变开始发生时的气体释放速率 会发生阶跃性变化. 论文采用主成分分析法(PCA)对挥发性物质的红外光谱进行了分类，可以准确地区分未变质、轻度 变质和重度变质的葡萄. 论文的结论说明挥发性物质的 FTIR 光谱分析可以有效鉴别陉藏中葡萄的劣变程度. 而且由于 挥发性气体在葡萄劣变中的阶跃变化性质，使这种鉴别方法具有不易受葡萄数量、存放方式影响的优点. 论文的研究 为葡萄劣变监测设备研制提供了理论和技术基础.
\end{abstract}

关键词＼cjkstart葡萄劣变；挥发性物质；傅里叶变换红外光谱; 主成分分析; 分类

\section{Analysis of Volatiles during Grape Deterioration Using FTIR}

\author{
Wang, Wenzhong $^{a, b} \quad$ Dong, Daming* ${ }^{*}$, Zheng, Wengang $^{a} \quad$ Han, Junfeng ${ }^{b}$ \\ Ye, Song ${ }^{b} \quad{\text { Jiao, } \text { Leizi }^{a} \quad \text { Zhao, Xiande }}^{a}$ \\ ( ${ }^{a}$ National Engineering Research Center for Information Technology in Agriculture, Beijing Academy of Agriculture and \\ Forestry Sciences, Beijing 100097) \\ $\left({ }^{b}\right.$ School of Electronic Engineering and Automation, Guilin University of Electronic Technology, Guilin 541004)
}

Abstract Grape is very perishable in transportation and storage, so its early warning is particularly important to lower the risks of large-scale deterioration. In order to study grape deterioration process, we analyzed the volatile compounds from grapes using Fourier transform infrared (FTIR) spectroscopy. Several grapes were put in the sample compartment of the FTIR spectrometer for $2 \mathrm{~h}$ per day. Then, the volatile compounds vaporized from the grapes were measured directly using the spectrometer. A high energy ceramic IR-source was used to improve the signal-to-noise ratio. We collected the FTIR spectrum before sample was put in as a background to eliminate the influence of air. Spectral signatures of the volatiles from grapes were analyzed and used to classify the grape samples into deterioration or not. By spectral analysis, the volatile mainly includes ethyl acetate, ethanol and carbon dioxide. The above three volatile vaporized more and more from the grapes during deterioration process. We also found that the release rates of volatile compounds get its highest value when the grapes just started deteriorating, so, this value could be used to monitor the beginning of deterioration. The methods to classify grapes deterioration levels were also studied. Firstly, grape deterioration processes were divided into three stages, fresh, slight deterioration and severe deterioration, by appearance and sensory evaluation. Then, a principle compounds analysis (PCA) was used for unsupervised classification to FTIR spectra. Results showed that this method could distinguish grapes into fresh and deterioration by choosing proper data pre-processing algorithms. This paper provides a new way to study the fruit deterioration mechanism, and premise a foundation for developing early-warning equipment for evaluation and monitoring fruit deterioration during its storage and transportation. Furthermore, because of the step change of release rates of volatile compounds at the beginning of deterioration, this kind of classifying method and monitoring system may not influenced by grapes quantity and store patterns.

Keywords grape deterioration; volatile compounds; Fourier transform infrared (FTIR); principal component analysis (PCA); classification

\section{1 引言}

葡萄是世界上栽种最广泛的果树之一，果实属浆
果. 由于葡萄果皮较薄, 含糖量高, 在运输和败藏过程 中容易发生腐烂 ${ }^{[1]}$. 因此，及早地对葡萄劣变程度进行

*E-mail: dongdm@nercita.org.cn; Tel.: 010-51503654; Fax: 010-51503626

Received November 5, 2012; published December 28, 2012.

Supporting information for this article is available free of charge via the Internet at http://sioc-journal.cn.

Project supported by the National Natural Science Foundation of China (Nos. 31101748, 31271614) and Innovation Building Program of Beijing Academy of Agriculture and Forestry Sciences (No. KJCX201102001).

项目受国家自然科学基金(No. 31101748, 31271614)和北京市农林科学院创新能力建设专项(No. KJCX201102001)资助. 
探测, 对将要发生、或少量腐败现象进行预警具有重要 的意义.

气味在表征食品新鲜度方面有着广泛的应用, 同时 具有快速、无损、实时等优点. 很多学者通过电子鼻系 统、气相色谱等分析工具对食品气味进行分析来判断食 品的新鲜度, 取得了良好的效果. 如 Papadopoulou ${ }^{[2]}$ 和 Barbri 等 ${ }^{[3]}$ 使用电子鼻系统对猪、牛、羊肉质量进行快 速检测, 对新鲜、腐烂的分类正确率均达到了 $80 \%$ 以上. Jorgensen ${ }^{[4]}$ 和 Wierda 等 ${ }^{[5]}$ 使用 GC-MS 对三文鱼在咜藏 过程中挥发物质进行分析, 表明 GC-MS 可用于对三文 鱼货架期的预测. 但电子鼻在实际应用中存在着易中 毒、交叉敏感等问题 ${ }^{[6]}$, 致使上述方法在工程实现中存 在一些缺陷.

傅里叶变换红外光谱是一种高效的未知气体测量 手段, 包括氮氧化物、醇类、碳氧化物等在内的多种气 态物质均在中红外区具有明显的指纹特征 ${ }^{[7,8]}$. 气体的 FTIR 光谱在很多领域都有着应用. 任晓宁等 ${ }^{[9]}$ 用快速扫 描 FTIR 光谱跟踪分析了奥克托金在不同气压和温度下 分解产物的种类和数量变化. Amamcharla 等 ${ }^{[10]}$ 建立了 牛肉沙门氏菌数量与挥发性物质 FTIR 光谱之间的关系, 表明 FTIR 光谱法能对牛肉是否被沙门氏菌感染进行表 征. 相对电子鼻、气相色谱等分析工具, 红外光谱法不 涉及化学反应, 很好地解决了传感器中毒和交叉串扰的 问题, 可不对气体进行处理而直接测量, 同时具有快 速、灵敏度高等优点 ${ }^{[11]}$, 更适于实现在线监测和预警. 李红雷等 ${ }^{[12]}$ 通过实验证明, 在 $10 \mathrm{~cm}$ 光程的气体池中, 3 $\mathrm{ppm}$ 浓度气体的 FTIR 光谱特征峰较为明显. 而水果变 质过程挥发的气体往往高于这个浓度. 因此, FTIR 光谱 法具有足够的灵敏度, 但目前尚未有 FTIR 光谱用于水 果劣变中气体分析的研究.

本文以 FTIR 光谱为技术手段, 测量并分析葡萄品 质劣变过程中所产生挥发性物质的光谱特征和规律. 利 用主成分分析方法对 FTIR 光谱进行分类研究, 从而为 葡萄品质劣变监测和预警提供科学依据.

\section{2 结果与讨论}

\section{1 劣变过程中的气体成分分析}

图 1 蓝色曲线是葡萄第 7 天(如图 2c, 已明显变质) 放置 $2 \mathrm{~h}$ 后挥发物质的 FTIR 光谱(吸光度), 红色曲线是 自然空气的光谱(气体池自然状态下放置 $2 \mathrm{~h}$ ). 表 1 统计 了参照 NIST 谱库分析的谱图中特征峰的归属.

由图 1 可见, 谱图中存在明显的水汽 $\left(\mathrm{H}_{2} \mathrm{O}\right)$ 和二氧 化碳 $\left(\mathrm{CO}_{2}\right)$ 特征. 空气中虽然也存在水汽和二氧化碳, 但自然空气光谱中 $\mathrm{H}_{2} \mathrm{O}$ 和 $\mathrm{CO}_{2}$ 的特征明显弱于葡萄挥 发物质中特征, 因此可知, 葡萄劣变后会挥发出大量的 水和二氧化碳. 根据前人的研究结果, 水和二氧化碳应 为葡萄果实呼吸作用 ${ }^{[13]}$ 和发酵 ${ }^{[14]}$ 过程中产生. 同时, 从 光谱特征中可以发现明显的乙酸乙酯 $\left(\mathrm{C}_{4} \mathrm{H}_{8} \mathrm{O}_{2}\right)$ 、乙醇
$\left(\mathrm{C}_{2} \mathrm{H}_{5} \mathrm{OH}\right)$ 特征，这与葡萄发酵过程中乙酸乙酯、乙醇占 挥发性物质主要成分的研究结论 ${ }^{[15]}$ 相吻合. 惠国华 ${ }^{[16]}$ 在试验中发现葡萄劣变气体对以下气体传感器均有响 应：乙醇、甲烷、一氧化碳、丁烷、液化石油气、液化 气传感器, 但从图 1 未发现烷类物质. 这可能是由于这 些物质浓度达不到检测阈值、特征峰被 $\mathrm{H}_{2} \mathrm{O}$ 和 $\mathrm{CO}_{2}$ 吸 收区覆盖等原因造成的. 但也有不存在上述气体的可 能，那么则说明上述气体的发现是电子鼻系统交叉敏感 问题所造成的，这有待后续试验验证.

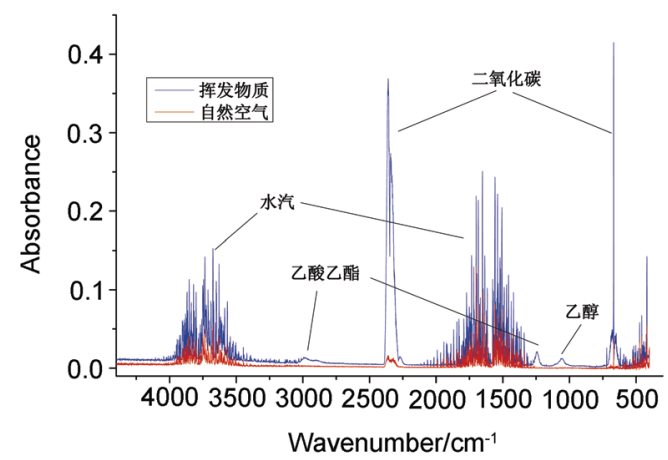

图 1 葡萄贮藏挥发性物质和自然空气的红外光谱

Figure 1 The FTIR spectrum of volatiles during grape storage and nature air

表 1 葡萄劣变气体谱图的谱峰归属 ${ }^{[17]}$

Table 1 The preliminary assignments of FTIR spectra of volatiles

\begin{tabular}{ll}
\hline Band $/ \mathrm{cm}^{-1}$ & Main attribution \\
\hline 1246,2997 & 乙酸乙酯 \\
1066 & 乙醇 \\
$600 \sim 730,2200 \sim 2400$ & 二氧化碳 \\
$1350 \sim 2300,3200 \sim 3600$ & 水汽 \\
\hline
\end{tabular}

\section{2 葡萄劣变过程和挥发性气体 FTIR 光谱关系分析}

\subsection{1葡萄劣变过程分级}

秦丹等将葡萄新鲜度分为了四个等级 ${ }^{[18]}$, 参考他 们的标准并结合实际情况, 我们将葡萄劣变过程分为未 变质、轻度变质、重度变质三个阶段, 分级标准如表 2 所示.

表 2 葡萄感官分级标准

Table 2 Standard of sensory evaluation on grapes

\begin{tabular}{llll}
\hline 劣变过程 & 外观 & 硬度 & 气味 \\
\hline 未变质 & 鲜绝有光泽 & 正常 & 正常的果香 \\
轻度变质 & 发暗、裂口出现霉菌 & 绵软 & 轻微的酸败味和酒精味 \\
重度变质 & 腐烂、严重脱水 & 软烂 & 强烈的酸败味和酒精味 \\
\hline
\end{tabular}

根据上述标准，本次试验中，葡萄在第 $1 \sim 2$ 天属于 新鲜, 3 4 天属于轻度变质, 5 7 天属于重度变质. 图 2 为第 1 天、第 4 天、第 7 天早上 9:00 的葡萄照片.

图 3 为第 1 天、第 4 天、第 7 天 11:00 时挥发物质 的 FTIR 吸光度谱. 可以看出, 葡萄在劣变前、中、后期 

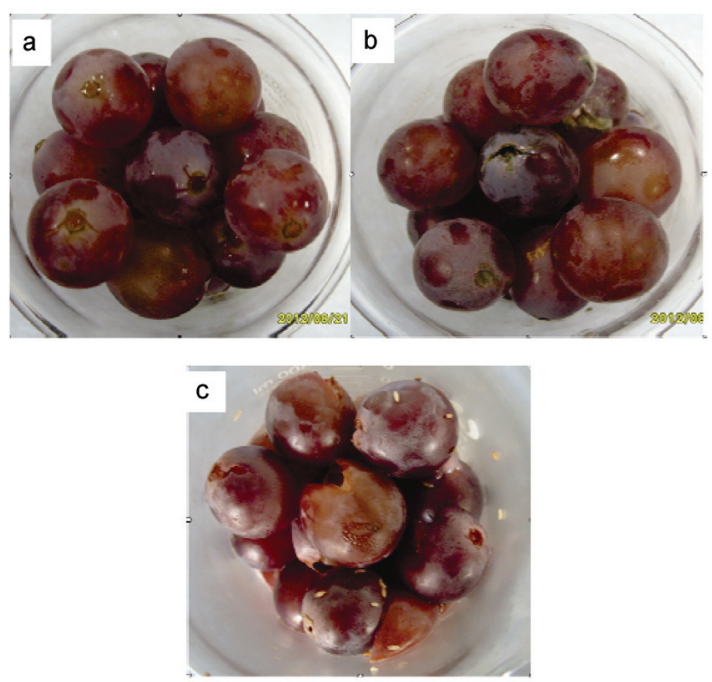

图 2 不同劣变时期葡萄照片. (a) 第 1 天, (b) 第 4 天, (c) 第 7 天

Figure 2 Grape pictures in different periods. (a)1st day, (b) 4th day, (c) 7 th day

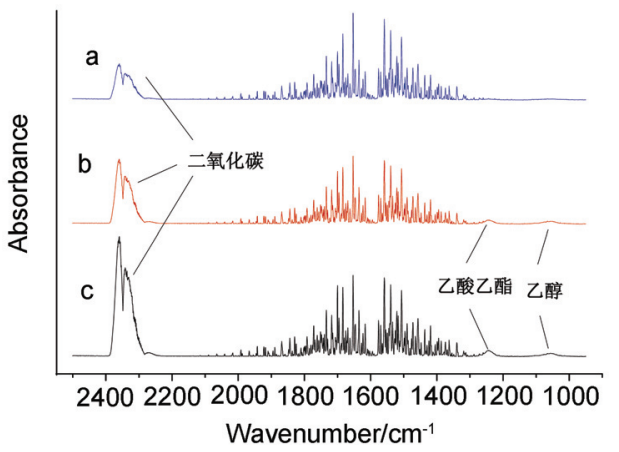

图 3 不同劣变时期典型红外光谱 $\left(2500 \sim 900 \mathrm{~cm}^{-1}\right)$. (a) 第 1 天, (b) 第 4 天, (c) 第 7 天

Figure 3 FTIR spectra of volatile compounds in different periods $\left(2500 \sim 900 \mathrm{~cm}^{-1}\right)$. (a) $1 \mathrm{st}$ day, (b) 4 th day, (c) 7 th day

$\mathrm{CO}_{2}$ 释放速度不断增加, 每个阶段相对上个阶段速度约 增加 1 倍. 这可能是由于前期仅为呼吸作用产生 $\mathrm{CO}_{2}$, 中、后期为呼吸作用和发酵作用两者共同产生. 在整个 实验阶段, 水分的挥发没有明显变化. 乙酸乙酯和乙醇 的特征峰在劣变前几乎观察不到, 到中期开始显现, 后 期比较明显, 后文将对劣变气体特征峰的吸光度进行量 化分析.

\subsection{2 劣变气体浓度变化趋势分析}

为了进一步分析劣变气体浓度与不同变质阶段的 关系, 对劣变气体的 FTIR 光谱特征峰进行分析, 从而 研究劣变气体浓度的变化规律. 根据朗伯-比尔定律, 在光程不变的情况下, 气体浓度与吸光度呈线性关系, 因而可以通过吸收峰的强弱找出劣变气体浓度与不同 劣变时期的相互关系. 一般来说, 可以通过计量吸收光 谱特征峰的峰高或者峰面积来计算气体的浓度 ${ }^{[19]}$. 图 4 和图 5 分别利用峰高法和峰面积法分析了挥发性物质光 谱特征在 $1 \sim 7 \mathrm{~d}$ 内的变化规律.
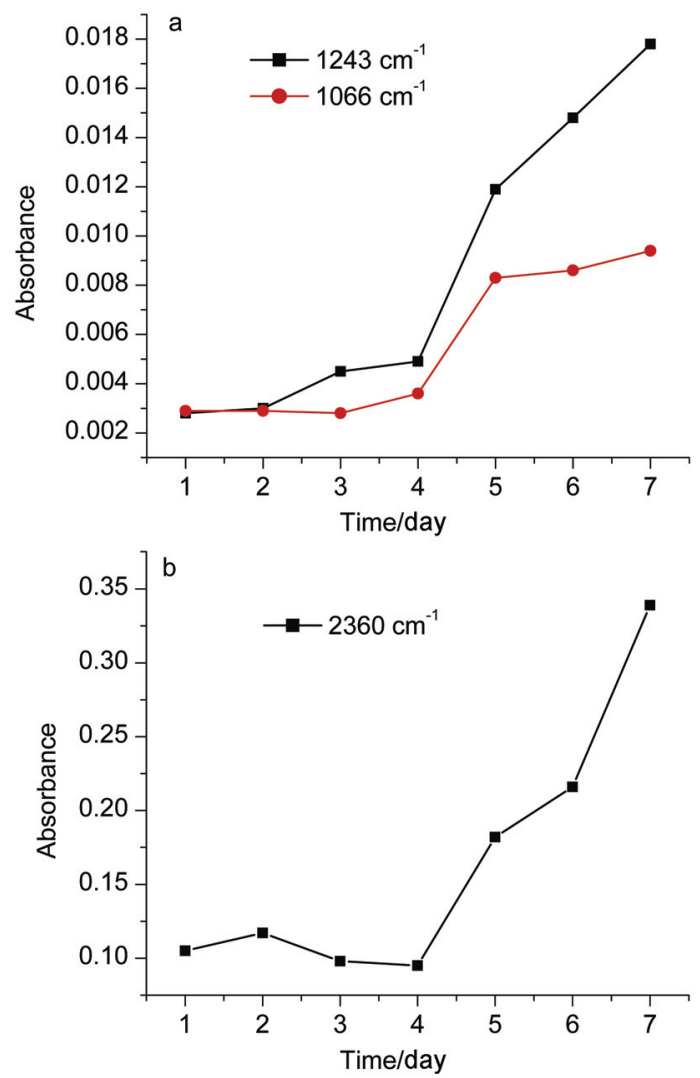

图 4 挥发性物质特征峰峰高随劣变的变化规律. (a) 乙酸乙酯、乙醇, (b) 二氧化碳

Figure 4 Changing laws of spectral peak height of volatile compounds during grape deterioration. (a) ethyl acetate, ethanol, (b) carbon dioxide

图 4a 和图 5a 为乙酸乙酯(1243 $\mathrm{cm}^{-1}$ )和乙醇(1066 $\left.\mathrm{cm}^{-1}\right)$ 光谱特征随䛎藏天数的变化规律. 从图中可以看 出, 峰高法和峰面积法的分析结果相近: 两种气体浓度 随劣变程度逐渐增加, 在整个劣变过程中有两个跃变, 第一个在劣变前进入劣变中期 $(2 \sim 3 \mathrm{~d})$, 乙酸乙酯浓度 提高了约 $75 \%$. 这可能是因为小部分葡萄裂口接触到葡 萄表皮上的白色粉晶(含野生酵母菌) $)^{[20]}$ ，同时受到表面 其他细菌感染，导致果肉开始发酵、变质，挥发性物质 释放速度开始加速; 第二个跃变是劣变中期进入劣变后 期(4 $5 \mathrm{~d})$, 乙酸乙酯释放速率增长了约 2 倍, 这可能是 因为葡萄的发酵和腐烂已经从表层进入葡萄内部，由个 别葡萄蔓延到多数葡萄, 劣变气体释放大大加速.

图 $4 \mathrm{a}$ 和图 $5 \mathrm{a}$ 为二氧化碳 $\left(2360 \mathrm{~cm}^{-1}\right)$ 光谱特征随咜 藏天数的变化规律. 从图中可以看出, 峰高法和峰面积 法的分析结果相近: $\mathrm{CO}_{2}$ 在劣变前、中期基本保持平稳, 甚至在第 3 天略有下降趋势. 中期到后期有剧烈变化. 这可能是因为葡萄浆果作为非呼吸跃变型果实, 在败藏 过程中呼吸强度基本一致 ${ }^{[21]}$, 而个别葡萄轻微的霉变 并不能对空气中的 $\mathrm{CO}_{2}$ 的浓度造成较大影响; 但是到劣 变中、后期，葡萄大面积腐烂、发酵，释放出大量 $\mathrm{CO}_{2}$, 因此监测到 $\mathrm{CO}_{2}$ 浓度剧增. 

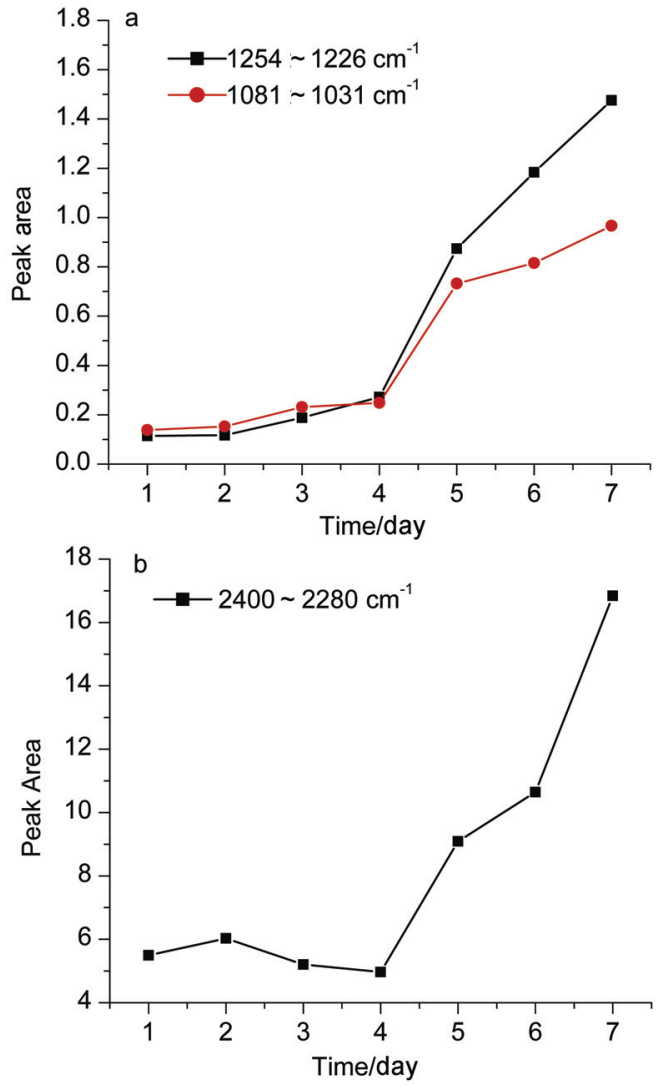

图 5 挥发性物质特征峰峰面积随劣变的变化规律. (a) 乙酸乙酯、乙 醇, (b) 二氧化碳

Figure 5 Changing laws of spectral peak area of volatile compounds during grape deterioration. (a) ethyl acetate, ethanol, (b) carbon dioxide

从乙酸乙酯、乙醇、二氧化碳的光谱变化规律可知, 三种气体在劣变过程中并不是稳态增加, 而是存在一个 阶跃的变化. 这种规律表明利用 FTIR 光谱分析葡萄劣 变时, 可以以气体浓度的跃变点为判断依据, 因而识别 方法受气体总排放的影响会比较小, 即不易受葡萄数 量、存放方式等因素的影响.

\section{3 基于 FTIR 光谱的葡萄劣变过程分类}

上述分析表明葡萄所挥发出气体的 FTIR 光谱随着 劣变程度加重具有明显的增加、跃迁等特征, 可以通过 某一种气体的排放量识别葡萄的劣变程度. 由于劣变过 程有多种气体的排放, 可以通过 PCA 等多元变量分析 的方法对劣变状态进行分析.

\subsection{1 数据预处理}

选择 $800 \sim 3200 \mathrm{~cm}^{-1}$ 范围为分析对象, 包含了二氧 化碳、水汽、乙酸乙酯和乙醇的特征峰. 由于测量过程 中气流干扰、基线漂移等问题的存在, 在使用化学计量 法建立模型之前, 对光谱进行预处理, 消除与光谱数据 无关的信息和噪声十分关键和必要 ${ }^{[22]}$. 使用 OPUS6.5 对光谱进行预处理, 包括对光谱进行归一化, 基线校正, 13 点平滑处理.

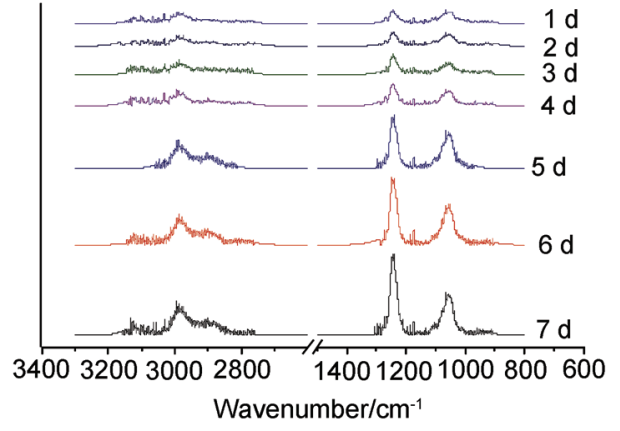

图 6 光谱预处理后的 1 $7 \mathrm{~d}$ 劣变气体吸光度谱图

Figure 6 Day $1 \sim 7$ FTIR spectra of volatile compounds after spectra pre-processing

\subsection{2 针对劣变气体光谱的主成分分析法分类}

为了研究葡萄败藏过程是否劣变的 FTIR 光谱分类 方法, 利用 PCA 对光谱进行了处理和分析. 主成分数取 为 4. 结果表明第一特征向量包含了二氧化碳、水汽、 乙酸乙酯和乙醇的特征峰, 第二特征向量主要表现在水 汽吸收带. 第一主成分对方差的贡献率为 $87 \%$, 第二主 成分对方差的贡献率为 $11 \%$. 图 7 表示了用前两个主成 分对葡萄劣变阶段分类的结果，由图可知，未变质、轻 度变质、重度变质可以在图中得到明显的区分. 进一步, 可以建立一个聚类分析的模型, 并对更多的实验结果进 行预测. 但由于本文的主要目的是讨论葡萄劣变中挥发 气体的 FTIR 光谱特征, 聚类和模式识别的研究结果将 在后续论文中介绍.

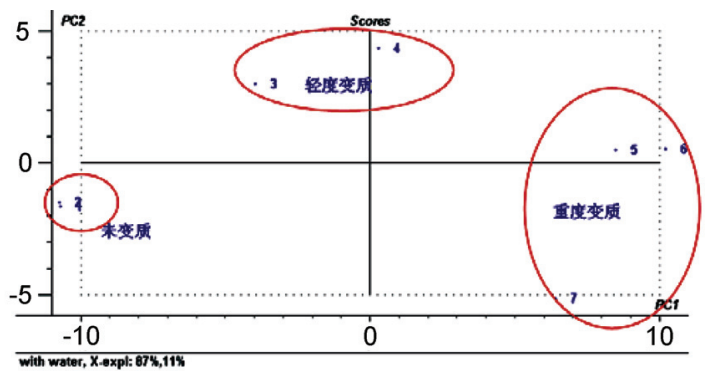

图 7 用 PCA 对葡萄劣变分类的结果

Figure 7 The classification results of grape deterioration using PCA

\section{3 结论}

本文采用傅里叶红外光谱仪研究了葡萄的劣变过 程, 对劣变过程中的挥发性物质进行了定性和定量的分 析, 结果表明 FTIR 方法可以对葡萄的劣变程度进行测 量, 这为水果腐败机理研究提供了一种新表征技术手 段. 该方法有快速、准确、简便等优点，其结果有望在 便携式和实时监测水果腐烂仪器上进行应用.

(1)葡萄在品质劣变过程中, 会释放出二氧化碳、乙 醇、乙酸乙酯、水汽等气体. 在自然状态、 $24 \mathrm{~cm}$ 光程 条件下利用主动式 FTIR 测量, 这些气体表现出了明显 的红外指纹图谱特征. 
(2)通过分析挥发性气体 FTIR 光谱的特征峰峰高、 峰面积等参数, 可以反映挥发气体的浓度变化规律: 气 体浓度随劣变呈现逐渐增强的趋势. 释放速率在未发生 劣变时基本保持平稳, 一旦少数葡萄发生劣变, 劣变气 体释放速率就会发生跃变. 通过监测这个跃变过程, 可 以实现对葡萄变质的预警，并消除葡萄数量、咜藏空间、 残留气体对预警准确度的影响.

(3)在挥发气体 FTIR 光谱基础上, 利用 PCA 方法对 劣变程度进行分类, 可以明显地区分未变质、轻度变质 和重度变质的葡萄样本.

(4)相对于其他劣变气体测量方法, 论文讨论的方 法具有很多优势. GC-MS 可以对劣变中的痕量气体进行 测量, 但依赖于复杂的取样、前处理过程, 缺乏实时性; 电子鼻阵列可以实时分析劣变气体, 但对环境的要求 高, 易中毒、有交叉敏感问题. FTIR 方法具有同时测量 多种组分、高灵敏、快速的优势. 更为重要的是, 经 FTIR 方法探索后, 可以将系统简化为特定波段光源和单元探 测器组成的简易系统, 专门针对某几种气体(如本文所 述的乙酸乙酯、乙醇等)进行测量和分析, 从而极大程度 降低成本.

\section{4 实验部分}

\section{1 实验材料}

供试材料为购自北京果多鲜超市当天采摘的巨峰 葡萄, 选取 3 个颗粒大小均匀、成熟度一致的果穗(不进 行清洗，以免洗掉表皮上的野生酵母菌).

\section{2 试验设备及参数设置}

实验采用 VERTEX 70 型 FTIR 光谱仪(德国 Bruker 公司). 仪器内置高能量、空气冷却 MIR/FIR 陶瓷光源; DTGS 探测器 $(7 \sim 14 \mu \mathrm{m})$; 在实验中, 光谱仪的光谱采 集范围设定为 $4000 \sim 400 \mathrm{~cm}^{-1}$, 分辨率 $0.5 \mathrm{~cm}^{-1}$, 扫描 频率为: $40 \mathrm{kHz}$, 扫描信号累加次数为 16 次. 仪器内气 体池的光程为 $24 \mathrm{~cm}$.

\section{3 试验方法和数据处理}

将葡萄取下 30 粒置入 $400 \mathrm{~mL}$ 烧杯中. 使用空气吹 扫光谱仪样品仓, 测量前使用 OPUS 6.5 软件(德国 Bruker 公司)采集背景. 每天早上 9:00 测量光谱仪样品 仓背景光谱, 然后将葡萄样品放入样品仓, 11:00 测量挥 发物质的 FTIR 光谱, 实验 7 天. 分别保存样本的原始光 谱、原始光谱与测量前背景之间的差谱、以测量前背景 为基准的吸光度谱. 实验方法如图 8 所示. 测量完后将 烧杯取出, 用保鲜膜封好, 置于阴凉处.

使用 OPUS6.5 对采集的红外光谱进行数据预处理, 预处理包括对光谱进行归一化, 基线校正, 13 点平滑处 理. 使用 Camo 公司的 The Unscrambler 9.7 的主成分分 析(PCA)功能对样本进行分类.

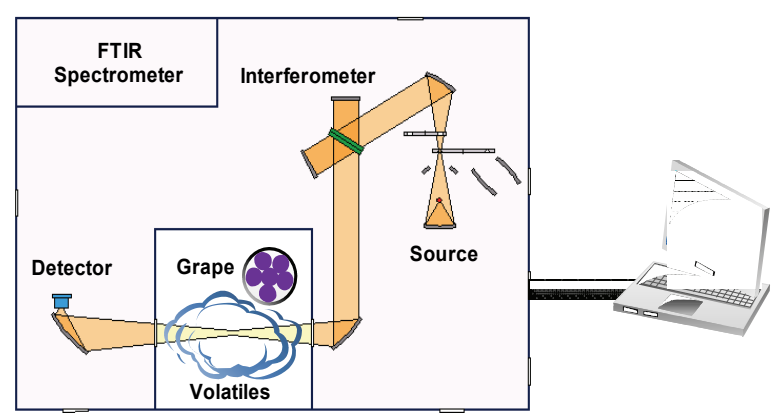

图 8 实验光路图

Figure 8 Experiment beam path diagram

\section{References}

[1] Li, G. F.; Liu, Y. S. J. Fruit Sci. 2000, 17, 63. (李桂芬，刘延松，果 树科学, 2000, 17, 63.)

[2] Papadopoulou, O. S.; Tassou, C. C.; L;.Schiavo, L.; Nychas, G. J.; Panagou, E. Z. Procedia Food Science 2011, 1, 2003.

[3] Barbri, N. E; Llobet, E.; El Bari, N. E.; Correig, X.; Bouchikhi, B. Sensors 2008, 8, 142 .

[4] Jorgensen, L. V.; Huss, H. H.; Dalgaard, P. J. Agric. Food Chem. 2001, 49(5), 2376.

[5] Wierda, R. L.; Fletcher, G.; Xu, L.; Dofou, J. J. Agric. Food Chem. 2006, 54(22), 8480 .

[6] Wilson, A. D. Procedia Technology 2012, 1, 453.

[7] Ferraro, J. R.; Basile, L. J. Fourier Transform Infrared Spectroscopy, Academic Press, New York, 1985, pp. 231 242 .

[8] Davidwt, G.; Williamg, M.; Michaelt, C.; Darolde, W.; Allen, R. Global Biomass Burning-Atmospheric, Climatic, and Biospheric Implications, MIT Press, Cambridge, MA, 1991, pp. 230 239.

[9] Ren, X. N.; Shao, Y. H.; Wang, X. H.; Zhao, F. Q.; Liu, Z. R.; Zhang, G.; Heng, S. Y.; Xie, M. Z. Acta Chim. Sinica 2010, 68(12), 1193. (任晓宁, 邵颖惠, 王晓红, 赵风起, 刘子如, 张自, 衡淑云, 谢明召, 化学学报, 2010, 68(12), 1193.)

[10] Amamcharla, J. K.; Panigrahi, S.; Logue, C. M.; Marchello, M.; Sherwood, J. S. Biosyst. Eng. 2010, 9, 107.

[11] Jiang, P. G. Anal. Instrum. 2009, (6), 77. (姜培源, 分析仪器, 2009, (6), 77.)

[12] Li, H. L.; Zhou, F. J.; Tan, K. X.; Gao, W. S. Automation of Electric Power Systems 2005, 29(18), 62. (李红雷, 周方洁, 谈克雄, 高文 胜, 电力系统自动化, 2005, 29(18), 62.)

[13] Liang, L. Y.; Hao, L. P.; Yan, S. J. Transactions of the CSAE 2003 , 19(4), 205. (梁丽雅, 郝利平, 问师杰, 农业工程学报, 2003, 19(4), 205.)

[14] Li, Y. F. Sino-Overseas Grapevine and Wine 2008, 2, 52. (李永峰, 中外葡萄和葡萄酒, 2008, 2, 52)

[15] Yang, Y.; Xu, Y. W.; Xue, J. X.; Liu, Y. L. Microbiology China 2004, 34(4), 757. (杨芗, 徐艳文, 薛军霞, 刘延林, 微生物学通 报, 2004, 34(4), 757.)

[16] Hui, G. H.; Li, P.; Wu, Y. L.; Zhu, L. S. Transactions of the CSAE 2012, 28(6) 264. (惠国华，厉鹏，吴玉玲，朱琳姗，农业工程学报， 2012, 28(6), 264.)

[17] Eds.: Linstrom, P. J.; Mallard, W. G., NIST Chemistry WebBook, NIST Standard Reference Database Number 69, National Institute of Standards and Technology, Gaithersburg MD, 20899, http://webbook.nist.gov, (retrieved October 27, 2012.)

[18] Xu, Y. W. M.S. Thesis, Northwest A\&F University, Xi'an, 2007. (徐 艳文，硕士论文，西北农林科技大学，西安, 2007.)

[19] Zhang, L.; Shao, S. Y.; Yang, L.; Dong, X. Q.; Ding, X. Q. Anal. Instrum. 2009, (2), 6. (张琳, 邵晟宇, 杨柳, 董晓强, 丁学全, 分析 仪器, 2009, (2), 6.)

[20] Ge, Y. Q.; Zhang, W. Y.; Chen, Y.; Ye, Q. Acta Horticulture Sinica 1997, 24, 120. (葛毅强, 张唯一, 陈颖, 叶强, 园艺学报, 1997, 24, 120.)

[21] Chu, X. L. Molecular Spectroscopy Analytical Technology Combined with Chemometrics and Its Application, Chemical Industry Press, Beijing, 2011, p. 41. (禇小立, 化学计量学方法与分子光谱 分析技术, 化学工业出版社, 北京, 2011, p. 41.)

[22] Xu, J. G.; Feng, X. L.; Guan, L.; Lei, M.; Yang, L. Journal of Logistical Engineering University 2011, 27(5), 51. (徐继刚, 冯新沪, 管 亮, 雷猛, 杨岗, 后勤工程学院学报, 2011, 27(5), 51.)

(Cheng, F.) 